mediate tumor necrosis factor alpha-converting, enzyme-dependent ectodomain shedding induced by phorbol myristate acetate. FASEB J. 15:303-305.

11. Cao, T.T., et al. 2005. Increased nuclear factorerythroid 2 p 45-related factor 2 activity protects SH-SY5Y cells against oxidative damage. J. Neurochem 95:406-417.

12. Itoh, K., et al. 1997. An Nrf2/small Maf heterodimer mediates the induction of phase II detoxifying enzyme genes through antioxidant response elements. Biochem. Biophys. Res. Commun. 236:313-322.

13. Lee, J.M., Chan, K., Kan, Y.W., and Johnson, J.A. 2004. Targeted disruption of Nrf2 causes regenerative immune-mediated hemolytic anemia. Proc. Natl. Acad. Sci. U. S. A. 101:9751-9756.
14. Chan, K., Han, X.D., and Kan, Y.W. 2001. An important function of $\mathrm{Nrf} 2$ in combating oxidative stress: detoxification of acetaminophen. Proc. Natl. Acad. Sci. U. S. A. 98:4611-4616.

15. McWhirter, S.M., et al. 2004. IFN-regulatory factor 3 -dependent gene expression is defective in Tbk1deficient mouse embryonic fibroblasts. Proc. Natl. Acad. Sci. U. S. A. 101:233-238.

16. Kolls, J.K., and Linden, A. 2004. Interleukin-17 family members and inflammation. Immunity. 21:467-476.

17. Langrish, C.L., et al. 2004. IL-12 and IL-23: master regulators of innate and adaptive immunity. Immunol. Rev. 202:96-105.

18. Rangasamy, T., et al. 2005. Disruption of Nrf2 enhances susceptibility to severe airway inflamma- tion and asthma in mice. J. Exp. Med. 202:47-59.

19. Paterson, R.L., Galley, H.F., and Webster, N.R. 2003. The effect of $\mathrm{N}$-acetylcysteine on nuclear factorkappa B activation, interleukin-6, interleukin-8, and intercellular adhesion molecule- 1 expression in patients with sepsis. Crit. Care Med. 31:2574-2578.

20. Spapen, H.D., Diltoer, M.W., Nguyen, D.N., Hendrickx, I., and Huyghens, L.P. 2005. Effects of Nacetylcysteine on microalbuminuria and organ failure in acute severe sepsis: results of a pilot study. Chest. 127:1413-1419.

21. Guidot, D.M., and Brown, L.A. 2000. Mitochondrial glutathione replacement restores surfactant synthesis and secretion in alveolar epithelial cells of ethanol-fed rats. Alcohol. Clin. Exp. Res. 24:1070-1076.

\title{
The alchemy of tendon repair: a primer for the (S)mad scientist
}

\author{
Dwight A. Towler ${ }^{1}$ and Richard H. Gelberman ${ }^{2}$
}

\author{
1Department of Medicine, Division of Bone and Mineral Diseases and \\ ${ }^{2}$ Department of Orthopedic Surgery, Washington University School of Medicine, St. Louis, Missouri, USA.
}

\begin{abstract}
During vertebrate development, mesenchymal progenitors capable of forming bone, cartilage, muscle, fat, or tendon arise from either neural crest or somitic mesoderm. Transcriptional programs that specify mesenchymal cell fates are initiated and modified by paracrine cues provided by TGF- $\beta$ superfamily members and mediated in part via the regulated assembly of Smadcontaining multiprotein transcription factor complexes. In this issue of the JCI, Hoffmann and colleagues have identified that Smad8 activation drives tendon formation from $\mathrm{C} 3 \mathrm{H} 10 \mathrm{~T} 1 / 2$ cells, a murine cell line that recapitulates many features of normal multipotent mesenchymal cells (see the related article beginning on page 940). Cells programmed to the tenocyte cell fate in vitro formed tenogenic grafts in vivo. These results add to the accumulating evidence that proliferating, multipotent mesenchymal progenitor cells can be programmed to yield multiple cell types - e.g., osteoblasts, myocytes, chondrocytes, and tenocytes - that may be useful in cell-based therapeutic approaches to musculoskeletal diseases.
\end{abstract}

Tendon and ligament injuries represent some of the most common musculoskeletal disorders that clinicians address daily, ranging from as mundane as a mild ankle sprain to the crippling effects of Achilles tendon rupture or flexor tendon injury in the hand (1). Severe tendon injuries are difficult to manage. Surgical repairs frequently do not fully restore function due to fibrous adhesions or failure arising from the mechanical demands placed on

Nonstandard abbreviations used: BMP, bone morphogenetic protein; GDF, growth differentiation factor; L+MH2, R-Smad linker plus MH2 domain; R-Smad, receptor-regulated Smad.

Conflict of interest: The authors have declared that no conflict of interest exists.

Citation for this article: J. Clin. Invest. 116:863-866 (2006). doi:10.1172/JCI28320. imperfect integrative healing at tendontendon or tendon-bone interfaces (1).

The tendon itself is a dense, regular connective tissue consisting primarily of type I collagen and the interspersed specialized mesenchymal cells known as tenocytes that are responsible for the maintenance of collagen structure, with avascular bundles of primary fibers invested by a thin layer of endotenon, a fine loose connective tissue sheath, to form fascicles (2) (Figure 1). Parallel arrays of fascicles are bundled together to form tendon by the epitenon, a layer contiguous with the endotenon through which the microvasculature, lymphatics, and innervation delicately traverses (2).

The ontogeny of the tenocyte lineage is only beginning to be understood. Elegant work recently forthcoming from the Tabin laboratory has demonstrated that during embryogenesis, a unique compartment of the somite called the syndetome provides tenocyte progenitors (3). Inductive interactions between the well-described myotome and sclerotome layers generate the syndetome, demarcated at the earliest stages of development by expression of the basic helix-loop-helix transcription factor gene Scleraxis. In addition to promoting tenocyte "birth," the myoprogenitors of the myotome appear to prevent chondrogenic differentiation of tenocyte progenitors. The paracrine signals that fine-tune multipotent mesenchymal progenitors to the unique tenocyte fate during normal development are virtually unknown, but appear to involve FGFs (3) and key members of the TGF- $\beta$ superfamily such as growth differentiation factor 5 (GDF5) and GDF7 (4).

\section{A tenogenic Smad}

Thus, given our limited understanding of how mesenchymal progenitors are efficiently recruited to the tenocyte lineage, the recent progress made by Hoffmann and colleagues in a report in this issue of the JCI is quite remarkable (5). Their insights into the mechanisms controlling tenocyte differentiation arose from fortuitous observations made while studying Smad signaling in C3H10T1/2 cells - a murine multipotent mesenchymal cell line that recapitulates many features of par- 


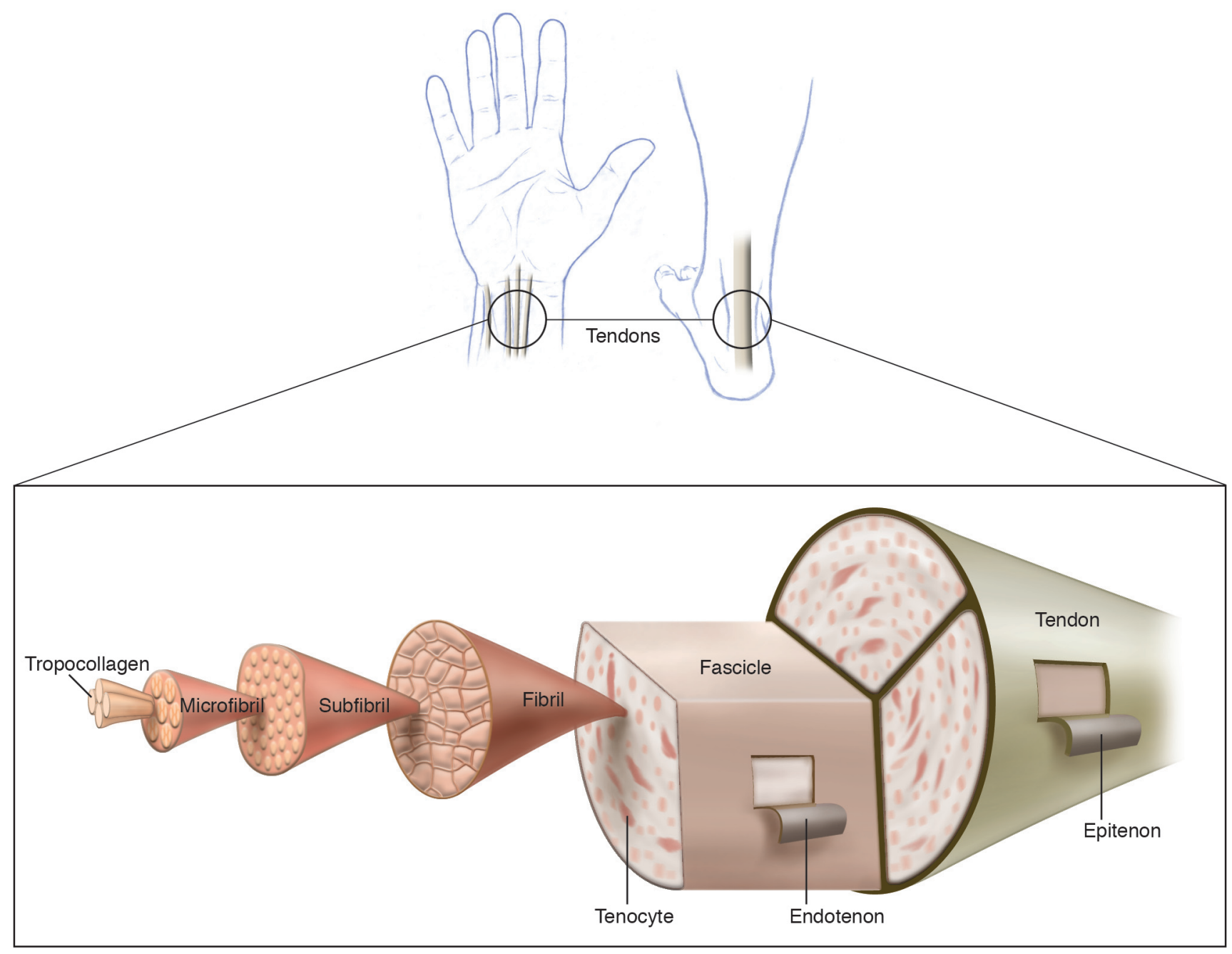

Figure 1

Organizational structure of tendon. Tendon is a nonmineralized, dense regular connective tissue that consists primarily of type I collagen. The major executive cell type of tendon, the tenocyte, synthesizes and secretes trihelical tropocollagen that is assembled and cross-linked in parallel fibrillar arrays. Higher-order organization of these arrays is provided by the endotenon, a loose connective tissue layer that envelopes collagen fibrils to form tendon fascicles. Fascicles in turn are bundled together by the epitenon, a layer contiguous with the endotenon through which the microvasculature traverses and provides nutrient supply $(1,2)$. Dependent upon anatomic venue, the tendon either may (e.g., hand flexor tendon) or may not (e.g., Achilles tendon) be housed within a synovial sheath (1,2).

axial mesenchymal progenitors and can form osteoblasts, chondrocytes, adipocytes, or skeletal myocytes in culture $(6,7)$ (Figure 2A). Smads are a group of related intracellular proteins that transmit TGF- $\beta$ superfamily signals from ligand-activated cell surface receptors to the nucleus. Smads are related to, but structurally distinct from, other intracellular effector proteins and are composed of 3 domains: an $\mathrm{N}$-terminal MH1 domain, a linker region, and a C-terminal MH2 domain (Figure 2B). Receptor-regulated Smads (R-Smads) associated with bone morphogenetic protein (BMP) signaling - Smad1, Smad5, and $\mathrm{Smad} 8$ - were expressed as consti- tutively active R-Smad linker plus MH2 domain ( $\mathrm{L}+\mathrm{MH} 2)$ fragments lacking the $\mathrm{N}$-terminal MH1 regulatory domain (8), and responses were assessed in the presence or absence of paracrine BMP2 signals (5). As previously shown (9), Smad1 and Smad5 directed alkaline phosphatase induction characteristic of osteoblast differentiation. By contrast, the Smad8 $\mathrm{L}+\mathrm{MH} 2$ fragment markedly enhanced the adoption of tenocyte-like cell morphology, suggesting tenocytic differentiation. Consistent with this notion, the syndetome and tenocyte marker Scleraxis (3) was induced by the BMP2/Smad8 L+MH2 combination along with other charac- teristic tenocyte genes such as Six1, Six2, EphA4, and procollagen type I, $\alpha 1$ (Col1A1) (5). The expression of most osteogenic markers (e.g., parathyroid hormone/parathyroid hormone-related protein receptor and alkaline phosphatase) was downregulated by the BMP2/Smad $8 \mathrm{~L}+\mathrm{MH} 2$ tenocytic trigger, although osteocalcin expression was not. Intriguingly BMP2, but not TGF- $\beta$ or GDF5, could provide paracrine cues necessary for efficient tenocyte differentiation directed by Smad8 L+MH2. Importantly, C3H10T1/2 cells engineered to express both BMP2 and Smad8 L+MH2 provided gap replacement of tendon-like tissue in a rat tenotomy model. Cells 
A

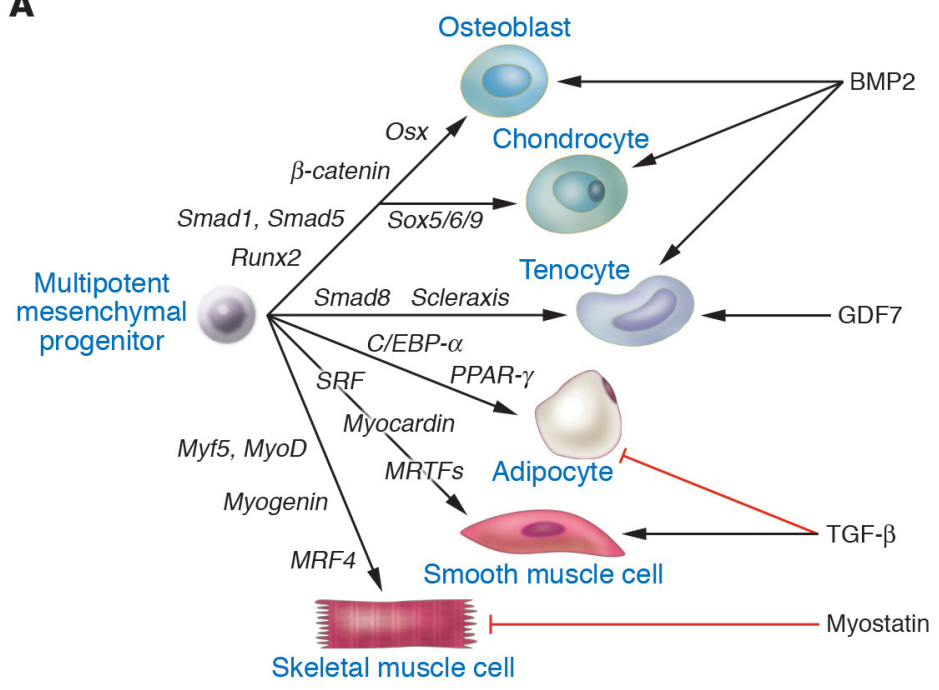

B

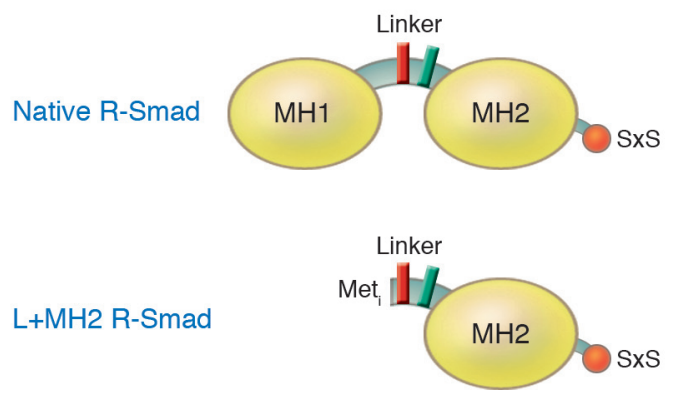

Figure 2

Lineage programming of mesenchymal progenitors: a working model. (A) During development, mesenchymal progenitors capable of forming bone, cartilage, muscle, fat, or tendon arise from either neural crest or from mesoderm. Transcriptional programs (shown in italics) that specify mesenchymal cell fates are initiated and modified by paracrine cues provided by TGF- $\beta$ superfamily members, mediated via regulated assembly of Smad-containing multiprotein transcription factor complexes (8). During somitic mesoderm lamination, the syndetome - demarcated by Scleraxis expression - forms at the interface between the bone-forming sclerotome and muscle-forming myotome to generate tenocytes of axial tendons (3). Similar mechanisms are likely deployed during appendicular tendon formation. Not shown are the influences of paracrine Wnt and FGF signaling cascades and homeodomain proteins of the Pax, Msx, DIx, and Six families that modulate BMP signaling. The data reported by Hoffmann et al. (5) indicate that activated Smad8 promotes tenocyte differentiation of C3H10T1/2 cells. Whether Smad8 participates in syndetome development is unknown. C/EBP, CCAAT/enhancer binding protein; MRF, muscle regulatory factor; MRTF, myocardin-related transcription factor; SRF, serum response factor. (B) R-Smad and L+MH2 structures. Although the MH1 domain encodes a DNA-binding function, Smad interaction with other transcription factors is required for gene regulation; these interactions are directed by the $\mathrm{L}+\mathrm{MH} 2$ domain (8). The SxS motif is the cognate for phosphorylation-dependent Smad activation by activin-like kinase receptors. Smad4 and importins mediate nuclear entry. Since the Smad8 L+MH2 fragment lacks intrinsic DNA binding, its tenogenic actions are mediated by regulatory protein-protein interactions.

expressing BMP2, Smad8 L+MH2, or the combination BMP2/Smad8 L+MH2 were introduced to a surgical partial-thickness Achilles tendon gap on an implantable collagen matrix sponge. Using histology and micro-MRI to monitor collagen fiber orientation and healing in vivo, the authors documented neotendon formation in implanted sponges carrying $\mathrm{C} 3 \mathrm{H} 10 \mathrm{~T} 1 / 2$ cells programmed with BMP2/Smad8 L+MH2. No ectopic endochondral bone formation occurred, and laser capture microdissection and RTPCR confirmed murine Col1A1 expression. By contrast, C3H10T1/2 cells programmed with only BMP2 generated ectopic bone and cartilage foci within the grafts, while Smad8 L $+\mathrm{MH} 2$ cells yielded only unorganized loose connective tissue. Immunohistochemistry to detect LacZ expression following adenovirus tagging and engraftment confirmed that the engineered BMP2/Smad8 L+MH2 cells did indeed contribute to the neotendon formed in vivo. Thus, the authors demonstrate the formation of tendon-like neotendon efficiently in vivo by BMP2 and Smad8 programming of mesenchymal progenitor cells (5).

\section{The combinatorial conundrum}

Many questions remain to be answered regarding the science of signal transduction and the needs of musculoskeletal medicine. It is unclear why paracrine BMP2 signaling is required to efficiently elaborate the full tenogenic potential of a constitutively active Smad8 L+MH2 fragment; it remains to be determined why removal of the MH1 domain - although capable of permitting Smad8 L+MH2 transactivation in transient transfection assays - is insufficient to promote robust tenogenesis in the absence of BMP2-generated signals. BMP2 receptor activation may trigger the phosphorylation-dependent nuclear transport and coregulator recruitment necessary for $S$ mad8 tenogenic transcriptional complexes (8). While BMP2 clearly alters the phosphorylation of Smad8, it cannot activate full-length Smad8 - i.e., the intact $\mathrm{MH} 1+\mathrm{L}+\mathrm{MH} 2$ molecule (Figure 2B) (5). Other regulatory receptor-ligand pairs presumably activate Smad8 in native context, perhaps mediated by a GDF family member other than GDF5 (4). GDF7 (also known as BMP12) has recently been shown to drive rhesus marrow mesenchymal cell differentiation to the tenocyte lineage in vitro (10). Of note, neither GDF5 nor TGF- $\beta 1$ were capable of enhancing Smad8 phosphorylation (5). Whether Smad8 participates in syndetome maturation and normal tendon development is as yet unknown. However, Smad8 signaling in mesenchymal cells is clearly not osteogenic and clearly drives gene expression programs different from those activated by Smad1 and Smad5. Indeed, Smad8 inhibits Smad1 and Smad5 signaling recruited by BMP4 during ventral mesoderm induction (11). While most artificially activated activinlike kinases can induce Smad8 phosphorylation, the "receptorology" of this R-Smad in vivo has yet to be determined. 


\section{A new opportunity in regenerative medicine}

Of note, BMP2 enhances collagen production by canine tenocytes (12) and has been used to enhance tendon-bone integration in canine disease models (13). In this robustly validated preclinical model, surgical technique and postoperative physical therapy can be used to assess functional outcome and biomechanical strength (1) - the hallmarks of tendon repair that must be addressed in future studies. Immunologic and vascularization responses that influence the formation of fibrous adhesions may also be augmented by programmed neotendon cells and thus hamper restoration of function (14). Given that multipotent mesenchymal progenitors resembling $\mathrm{C} 3 \mathrm{H} 10 \mathrm{~T} 1 / 2$ cells can be isolated from tissues such as adipose (15) and skeletal muscle (16), engineered autografts might be best explored in canine models of tendon injury repair to minimize the deleterious immune responses; such responses may limit healing, biomechanical strength, and restoration of function. Nevertheless, the novel results reported here by Hoffmann et al. (5) add to the accumulating evidence that proliferating, multipotent mesenchymal progenitor cells can be programmed to yield yet another cell type
- the tenocyte - which may be potentially useful in cell-based therapeutic approaches to musculoskeletal injuries (1).

Address correspondence to: Dwight A. Towler, Department of Internal Medicine, Division of Bone and Mineral Diseases, Washington University School of Medicine, Barnes-Jewish North Campus Box 8301, 660 South Euclid Avenue, St. Louis, Missouri 63110, USA. Phone: (314) 454-7434; Fax: (314) 454-8434; E-mail: dtowler@im.wustl.edu.

1. Boyer, M.I., Goldfarb, C.A., and Gelberman, R.H. 2005. Recent progress in flexor tendon healing. The modulation of tendon healing with rehabilitation variables. J. Hand Ther. 18:80-85; quiz 86.

2. Fenwick, S.A., Hazleman, B.L., and Riley, G.P. 2002. The vasculature and its role in the damaged and healing tendon. Arthritis Res. 4:252-260.

3. Brent, A.E., Schweitzer, R., and Tabin, C.J. 2003. A somitic compartment of tendon progenitors. Cell. 113:235-248

4. Wolfman, N.M., et al. 1997. Ectopic induction of tendon and ligament in rats by growth and differentiation factors 5,6 , and 7 , members of the TGF- $\beta$ gene family. J. Clin. Invest. 100:321-330.

5. Hoffmann, A., et al. 2006. Neotendon formation induced by manipulation of the Smad8 signalling pathway in mesenchymal stem cells. J. Clin. Invest. 116:940-952. doi:10.1172/JCI22689.

6. Cheng, S.L., Shao, J.S., Charlton-Kachigian, N., Loewy, A.P., and Towler, D.A. 2003. MSX2 promotes osteogenesis and suppresses adipogenic differentiation of multipotent mesenchymal progenitors. J. Biol. Chem. 278:45969-45977.

7. Jasuja, R., et al. 2005. Tetrahydrogestrinone is an androgenic steroid that stimulates androgen receptor-mediated, myogenic differentiation in C3H10T1/2 multipotent mesenchymal cells and promotes muscle accretion in orchidectomized male rats. Endocrinology. 146:4472-4478.

8. Massague, J., Seoane, J., and Wotton, D. 2005. Smad transcription factors. Genes Dev. 19:2783-2810.

9. Yamamoto, N., et al. 1997. Smad1 and smad5 act downstream of intracellular signalings of BMP-2 that inhibits myogenic differentiation and induces osteoblast differentiation in $\mathrm{C} 2 \mathrm{C} 12$ myoblasts. Biochem. Biophys. Res. Commun. 238:574-580.

10. Wang, Q.W., Chen, Z.L., and Piao, Y.J. 2005. Mesenchymal stem cells differentiate into tenocytes by bone morphogenetic protein (BMP) 12 gene transfer. J. Biosci. Bioeng. 100:418-422.

11. Nakayama, T., et al. 1998. Xenopus Smad8 acts downstream of BMP-4 to modulate its activity during vertebrate embryonic patterning. Development. 125:857-867.

12. Thomopoulos, S., Harwood, F.L., Silva, M.J., Amiel, D., and Gelberman, R.H. 2005. Effect of several growth factors on canine flexor tendon fibroblast proliferation and collagen synthesis in vitro. J. Hand Surg. (Am.). 30:441-447.

13. Martinek, V., et al. 2002. Enhancement of tendon-bone integration of anterior cruciate ligament grafts with bone morphogenetic protein-2 gene transfer: a histological and biomechanical study. J. Bone Joint Surg. Am. 84:1123-1131.

14. Bidder, M., Towler, D.A., Gelberman, R.H., and Boyer, M.I. 2000. Expression of mRNA for vascular endothelial growth factor at the repair site of healing canine flexor tendon. J. Orthop. Res. 18:247-252.

15. Zuk, P.A., et al. 2002. Human adipose tissue is a source of multipotent stem cells. Mol. Biol. Cell. 13:4279-4295.

16. Lee, J.Y., et al. 2000. Clonal isolation of musclederived cells capable of enhancing muscle regeneration and bone healing. J. Cell Biol. 150:1085-1100.

\title{
A new cardiac MASTer switch for the renin-angiotensin system
}

\author{
Thu H. Le and Thomas M. Coffman
}

Division of Nephrology, Department of Medicine, Duke University and Durham VA Medical Centers, Durham, North Carolina, USA.

\begin{abstract}
The aspartyl protease renin was first isolated from the kidney by Tigerstedt more than a century ago. In the kidney, renin secretion is tightly linked to sodium intake and renal perfusion pressure, reflecting the important role of the renin-angiotensin system (RAS) in controlling body fluid volume and blood pressure. The study by Mackins et al. in this issue of the JCI describes a novel source of renin: the mast cell (see the related article beginning on page 1063). This discovery suggests a distinct pathway for activation of the RAS that may have a particular impact on the pathogenesis of chronic tissue injury as well as more acute pathology such as arrhythmias in the heart.
\end{abstract}

Nonstandard abbreviations used: ACE, angiotensinconverting enzyme; JG, juxtaglomerular; RAS, reninangiotensin system.

Conflict of interest: The authors have declared that no conflict of interest exists.

Citation for this article: J. Clin. Invest. 116:866-869 (2006). doi:10.1172/JCI28312.

\section{Separate renin-angiotensin systems} in the circulation and in tissues

The renin-angiotensin system (RAS) is a hormone system in which the substrate protein angiotensinogen is sequentially cleaved by peptidases, renin and angiotensin-converting enzyme (ACE), to form the biologically active octapeptide angiotensin II (Figure 1). A substantial excess of angiotensinogen is present in serum, and ACE is ubiquitous in the endothelium and plasma (1). Accordingly, in the bloodstream, the amount of renin is the rate-limiting step determining the level of angiotensin II and thus the activity of the system.

The primary source of renin in the circulation is the kidney, where its expression and secretion are tightly regulated at the juxtaglomerular (JG) apparatus by 2 distinct mechanisms: a renal baroreceptor $(2,3)$ and sodium chloride delivery to the macula densa $(4,5)$. Through these sensing mechanisms, levels of renin in plasma can be incrementally titrated in response 